\title{
Evapotranspiração e coeficiente de cultura do algodoeiro irrigado a partir de imagens de sensores orbitais ${ }^{1}$
}

\author{
Cotton evapotranspiration and crop coefficient obtained by satellite images
}

\author{
Marcus Vinícius Cândido Bezerra ${ }^{2 *}$, Bernardo Barbosa da Silva², Bergson Guedes Bezerra ${ }^{2}$, Valéria Peixoto \\ Borges $^{3}$ e Aureo Silva de Oliveira ${ }^{4}$
}

\begin{abstract}
Resumo - O presente estudo teve como objetivos estimar a evapotranspiração - ETc e determinar a curva do coeficiente de cultura - Kc do algodoeiro irrigado através do Surface Energy Balance Algorithm for Land - SEBAL com imagens orbitais TM - Landsat 5. Foram utilizadas oito imagens distribuídas ao longo do ciclo fenológico do algodoeiro cultivado na Fazenda Busato localiza no município de Bom Jesus da Lapa, região do Médio São Francisco, Estado da Bahia $\left(13^{\circ} 15^{\prime} 18^{\prime}\right.$ 'S, $43^{\circ} 25^{\prime} 05^{\prime}$ 'W, 436 m). A classificação climática da região segundo Köppen é BSwh'. O saldo de radiação foi calculado a partir de imagens da temperatura, emissividade da superfície, índices de vegetação, albedo e calculados os fluxos de calor no solo e sensível para obter-se o fluxo de calor latente e a ETc. Verificou-se que o índice de vegetação NDVI apresentou evolução concomitante com o ciclo da cultura, com valores máximos $(0,80)$ aos 70 dias após semeadura - DAS. A ETc e o Kc obtidos foram, respectivamente: 1,0 a 5,0 $\mathrm{mm} \mathrm{dia}^{-1}$ e 0,65 no período de desenvolvimento ( 7 e $70 \mathrm{DAS}$ ); $>6 \mathrm{~mm} \mathrm{dia}^{-1}$ e 1,18 durante a floração e formação dos capulhos e $2 \mathrm{~mm} \mathrm{dia}^{-1}$ e 0,66 no fim do ciclo. Os resultados mostram que o NDVI é um bom indicador do desenvolvimento do algodoeiro e os dados de ETc e Kc estão coerentes com relatos na literatura.
\end{abstract}

Palavras-chave - Algodão. Cultivo. Evapotranspiração.

\begin{abstract}
This research aimed determine cotton evapotranspiration - ETc and crop coefficient - Kc slope using the Surface Energy balance Algorithm for Land - SEBAL with TM-Landsat 5 images. We used eight images distributed throughout the cotton growth season on the Busato Farm located in Bom Jesus da Lapa, Médio São Francisco region, Bahia state (1315'18” S, $43^{\circ} 25^{\prime} 05^{\prime}$ ' W, $\left.436 \mathrm{~m}\right)$. The Climate classification of region by Köppen is BSwh'. The net radiation was calculated from surface temperature, surface emissivity, vegetation index and albedo imagesn and calculated soil and sensible heats fluxes to obtain the latent heat flux and ETc. The NDVI vegetation index presented concurrent development with the crop season, with maximum values (0.80) 70 days after sowing - DAS. Values of Etc and Kc were, respectively, 0 a $5.0 \mathrm{~mm} \mathrm{day}^{-1}$ e 0.65 in vegetation development period (7 e 70 DAS); > $6 \mathrm{~mm}_{\text {day }}{ }^{-1}$ e 1.18 during flowering and cotton bolls generation; and $2 \mathrm{~mm} \mathrm{day}^{-1}$ e 0.66 in the crop cycle ending. Results show that NDVI is a good indicator of the development of cotton and ETc and Kc data are consistent with reports in the literature.
\end{abstract}

Key words - Cotton. Crop. Evapotranspiration.

\footnotetext{
* Autor para correspondência

${ }^{1}$ Recebido para publicação 15/12/2010; aprovado em 14/06/2011

Extraído da Tese do primeiro autor, apresentada ao Programa de Pós-Graduação em Meteorologia, UACA/UFCG

${ }^{2}$ Universidade Federal de Campina Grande/UFCG, Unidade Acadêmica de Ciências Atmosféricas, Av. Aprígio Veloso, 882, Bodocongó, Campina Grande-PB, Brasil, 58.109-970, markusvynycyus@yahoo.com.br, bernardo@dca.ufcg.edu.br, bergson_bezerra@yahoo.com.br

${ }^{3}$ Bolsista do CNPq, Programa de Pós-Graduação em Meteorologia com área de concentração em Agrometeorologia, Universidade Federal de Campina Grande/UFCG, Unidade Acadêmica de Ciências Atmosféricas, Campina Grande-PB, Brasil, valpborges@gmail.com

${ }^{4}$ Universidade Federal do Recôncavo da Bahia/UFRB, Núcleo de Engenharia de Água e Solo, Via de acesso s/n, 44380-000, Cruz das Almas-BA, Brasil, aureo@ufba.br
} 


\section{Introdução}

Estudos hidrológicos em escala global têm indicado que no futuro se configurarão cenários de acentuados aumentos da demanda hídrica para atender as necessidades dos setores urbano, industrial e ambiental (FERERES; SORIANO, 2007). Assim, o uso eficiente da água em qualquer que seja a atividade, até mesmo doméstica, vem se tornando uma necessidade imprescindível.

Um dos setores onde o uso eficiente da água tem grande relevância é a agricultura irrigada, visto que a mesma é a maior usuária de água em escala global, atingindo porções superiores a 70\% do total disponível em áreas áridas e/ou semiáridas (FERERES; SORIANO, 2007; PERRY et al., 2009). Outro fato relevante que torna o uso eficiente da água na irrigação é o aumento da população e o consequente aumento da demanda por alimentos, uma vez que mais de $40 \%$ da produção mundial de alimentos advém da agricultura irrigada (FERERES; SORIANO, 2007).

Nas regiões semiáridas, a exemplo do Nordeste Brasileiro, a suplementação de água via irrigação é vital para produção de alimentos visto que as precipitações pluviométricas são irregulares e mal distribuídas. Atento a essa problemática surge a necessidade da adoção de práticas de manejo de irrigação a fim de garantir a sustentabilidade agrícola. Nesse contexto a determinação da evapotranspiração de culturas (ETc) torna-se de extrema importância.

A ETc pode ser determinada com precisão a partir dos métodos micrometeorológicos, lisímetros, balanço de água no solo, dentre outros métodos diretos. Por sua vez, o método indireto que usa o coeficiente de cultura, minuciosamente descrito pelo boletim da FAO número 56 (ALLEN et al., 1998), trata-se de uma metodologia eficiente na determinação da ETc e tem sido amplamente aplicado (ALLEN et al., 2005; ALLEN; PEREIRA, 2009; HUNSAKER et al., 2003; PEREIRA et al., 2009). O procedimento da FAO tem a seu favor a vantagem de não ser oneroso, pois não requer a instalação de aparatos experimentais, sendo necessários somente dados meteorológicos diários, disponível em estações meteorológicas para estimar a ET para uma condição de referência, a qual é multiplicada por um coeficiente de cultura. No entanto, o referido método apresenta uma limitação quanto à disponibilidade dos dados das variáveis meteorológicas necessárias para o cálculo da ETo. Nesse caso, a aplicação do mesmo é inviabilizada.

O Kc é basicamente a taxa da ETc presente na ETo, e representa uma integração dos efeitos das quatro principais características que distinguem uma cultura qualquer de uma cultura de referência, quais sejam a altura da planta, o albedo, a resistência estomática e a evaporação do solo, especificamente, o solo exposto (ALLEN et al., 1998). Além dos dados meteorológicos também se faz necessária a identificação do estágio de desenvolvimento da cultura e o conhecimento dos respectivos valores do Kc.

Tem sido verificado que o padrão sazonal do Kc apresenta similaridades com o comportamento sazonal dos índices de vegetação, a exemplo do NDVI (LÓPEZURREA et al., 2009). Com base nessas similaridades, a ETc de inúmeras culturas tem sido determinada com base na relação entre o NDVI e Kc (HUNSAKER et al., 2003, 2007; LÓPEZ-URREA et al., 2009).

Existem ainda os métodos baseados em medidas radiométricas obtidas a partir de sensores remotos tais como os sensores a bordo de satélites. Um desses métodos é o SEBAL - Surface Energy Balance Algorithm for Land (BASTIAANSSEN, 2000) e a sua versão aprimorada METRIC - Mapping Evapotranspiration at high Resolution and with Internalized Calibration (ALLEN et al., 2007a, 2007b) que têm sido amplamente aplicadas em diferentes regiões do planeta. $\mathrm{O}$ desempenho desses métodos tem sido testado em diferentes ecossistemas e regiões do planeta a exemplo dos Estados Unidos (ALLEN et al., 2007a, 2007b; BASTIAANSSEN et al., 2005), Brasil (BEZERRA et al., 2008) e China (LI et al., 2008). Em todos esses estudos o SEBAL e o METRIC tem alcançado desempenho satisfatório, apresentando diferenças, quando comparado com observações de campo, dentro da faixa das imprecisões instrumentais, lhe conferindo assim, robustez e confiabilidade suficientes para serem aplicados em escala operacional na determinação da ETc.

Diante do exposto, o presente estudo tem como objetivo estimar a ETc e determinar a curva do coeficiente de cultura do algodoeiro irrigado (cultivares Delta Penta e Delta Opal) através da aplicação do SEBAL com imagens TM - Landsat 5 ao longo do ciclo fenológico da cultura.

\section{Material e métodos}

A área de estudo compreende a Fazenda Busato localizada no município de Bom Jesus da Lapa $\left(13^{\circ} 15^{\prime} 18^{\prime}\right.$ ' S, 432 $5^{\prime} 05^{\prime}$ ' $\left.\mathrm{W}, 436 \mathrm{~m}\right)$ na região do Médio São Francisco, oeste do Estado da Bahia (FIG.1).

A classificação climática da região segundo Köppen é BSwh' caracterizado por semiárido quente, com chuvas de verão e período seco bem definido no inverno, e ausência de excedente hídrico. As médias anuais de temperatura, precipitação e evapotranspiração potencial são respectivamente $25,3{ }^{\circ} \mathrm{C}, 831 \mathrm{~mm}$ e 1.418 $\mathrm{mm}$. Na região predominam Latossolos e Cambissolos, com vegetação de contato caatinga-floresta estacional, conforme descrito por Borges et al. (2010). 


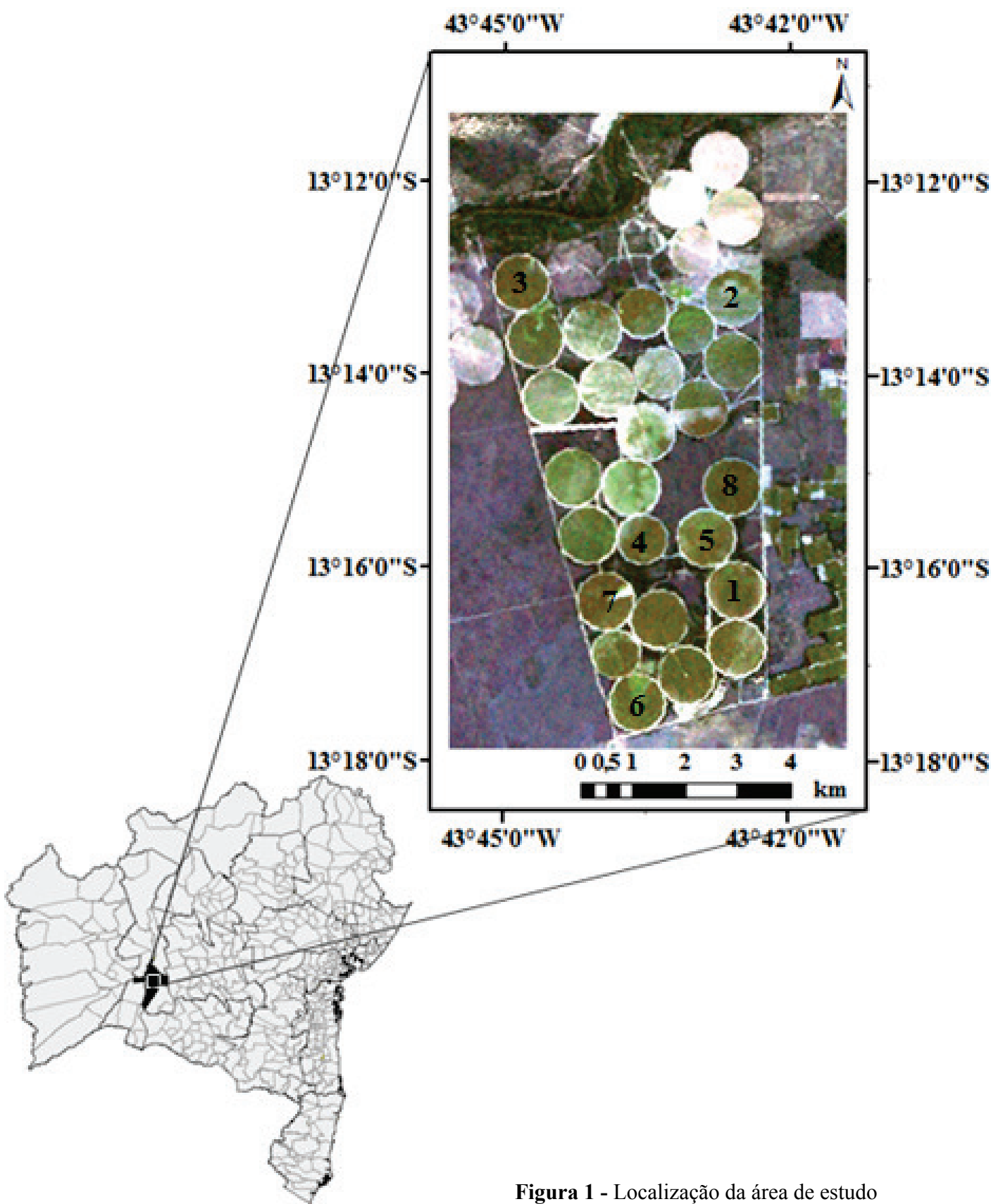

No período que compreendeu todo o ciclo da cultura do algodoeiro da área estudada, entre os meses de janeiro a agosto de 2007, foram registrados pela estação meteorológica automática local, ocorrência de precipitação total de $392 \mathrm{~mm}$, com máxima em fevereiro (287 mm), não ocorrendo precipitação entre maio e agosto de 2007. O mês mais quente foi janeiro, com média de $28,2{ }^{\circ} \mathrm{C}$ e o mês mais frio foi julho, com média de $23,8^{\circ} \mathrm{C}$ e temperatura média do período de $25,8^{\circ} \mathrm{C}$. Por sua vez, a radiação global média do período foi de $20 \mathrm{MJ} \mathrm{m}^{-2} \mathrm{dia}^{-1}$.
Figura 1 - Localização da área de estudo

As imagens de satélite utilizadas foram geradas pelo TM - Landsat 5, órbita 219 e ponto 69, adquiridas junto ao INPE (Instituto Nacional de Pesquisas Espaciais). Foram utilizadas oito imagens, obtidas em datas distintas do ciclo do algodoeiro: 20 de janeiro, 25 de março, 26 de abril, 12 de maio, 28 de maio, 13 de junho, 15 e 31 de julho de 2007. O satélite tem resolução temporal de 16 dias e espacial de $30 \mathrm{~m}$ e o horário de passagem sobre a área de estudo ocorreu, em todas as imagens, em torno das 10 horas. 
A ETc foi calculada a partir do fluxo de calor latente instantâneo - LE ( $\mathrm{W} \mathrm{m}^{-2}$ ), que por sua vez foi obtido como resíduo da equação do balanço de energia à superfície:

$\mathrm{LE}=\mathrm{Rn}-\mathrm{G}-\mathrm{H}$

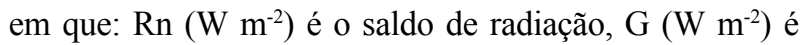
o fluxo de calor no solo e $\mathrm{H}\left(\mathrm{W} \mathrm{m}^{-2}\right)$ é o fluxo de calor sensível.

Inicialmente foi obtido o Rn pela equação seguinte, cujos procedimentos de cálculo de $\alpha, \mathrm{R}_{\mathrm{L} \downarrow}, \mathrm{R}_{\mathrm{L} \uparrow}$ e $\varepsilon_{\mathrm{o}}$ estão detalhadamente descritos em Allen et al. (2007a).

$$
\mathrm{Rn}=(1-\alpha) \mathrm{R}_{\mathrm{S} \downarrow}+\mathrm{R}_{\mathrm{L} \downarrow}-\mathrm{R}_{\mathrm{L} \uparrow}-\left(1-\varepsilon_{\mathrm{o}}\right) \mathrm{R}_{\mathrm{L} \downarrow}
$$

em que: $\mathrm{R}_{\mathrm{S} \downarrow}\left(\mathrm{W} \mathrm{m}^{-2}\right)$ é a radiação global instantânea no momento da geração da imagem medida na estação meteorológica, $\alpha$ é o albedo da superfície, $\mathrm{R}_{\mathrm{L} \downarrow}\left(\mathrm{W} \mathrm{m}^{-2}\right)$ é a radiação de onda longa emitida pela atmosfera, $\mathrm{R}_{\mathrm{L} \uparrow}\left(\mathrm{W} \mathrm{m}^{-2}\right)$ é a radiação de onda longa emitida pela superfície e $\varepsilon_{\mathrm{o}}$ é a emissividade da superfície.

O fluxo de calor no solo foi determinado conforme sugerido por Allen et al. (2007a):

$$
\begin{aligned}
& \mathrm{G}=[0,05+0,18 \exp (-0,521 . \mathrm{IAF}) \mathrm{Rn}](\mathrm{IAF} \geq 0,5) \\
& \mathrm{G}=[(1,80 \mathrm{Ts} / \mathrm{Rn})+0,084] \mathrm{Rn}(\mathrm{IAF}<0,5)
\end{aligned}
$$

em que: IAF é o Índice de Área Foliar e Ts é a temperatura da superfície $\left({ }^{\circ} \mathrm{C}\right)$.

Para o fluxo de calor em corpos d'água, cujo Índice de Vegetação da Diferença Normalizada (Normalized Difference Vegetation Index - NDVI) (Equação 5) é inferior a zero, assumiu-se que $\mathrm{G}$ equivale a $30 \%$ do Rn. Esse valor foi adotado para as condições dos copos de água presentes na cena, os quais são rasos e turvos.

$$
\mathrm{NDVI}=\frac{\rho_{4}-\rho_{3}}{\rho_{4}+\rho_{3}}
$$

em que: $\rho_{4}$ é a refletância da banda 4 e $\rho_{3}$ é a refletância da banda 3 do TM - Landsat 5.

Os valores de $\mathrm{H}$ foram estimados com base na velocidade do vento e na diferença de temperatura (dT) próxima à superfície entre os níveis de $0,1 \mathrm{~m}$ e $2,0 \mathrm{~m}$, segundo Allen et al. (2007a):

$$
H=c_{p} \rho \frac{d T}{r_{a h}}
$$

em que: $\rho$ é a densidade do $\operatorname{ar}\left(\mathrm{kg} \mathrm{m}^{-3}\right)$, $\mathrm{c}_{\mathrm{p}}$ é o calor específico do ar à pressão constante $\left(1.004 \mathrm{~J} \mathrm{~kg}^{-1} \mathrm{~K}^{-1}\right)$, a e b são constantes de calibração da diferença de temperatura,
Ts é a temperatura da superfície $(\mathrm{K})$ e $\mathrm{r}_{\mathrm{ah}}$ é a resistência aerodinâmica ao transporte de calor sensível $\left(\mathrm{s} \mathrm{m}^{-1}\right)$.

A principal hipótese do algoritmo SEBAL é considerar que existe uma relação linear entre a diferença de temperatura do ar e a temperatura da superfície, ou seja, $\mathrm{dT}=\mathrm{a}+\mathrm{b}$.Ts. A determinação dos coeficientes "a" e "b" requer a definição de dois pixels de referência (pixels âncoras) que representam condições extremas de temperatura e umidade, denominados, pixels quente e frio. O pixel frio localiza-se em uma área bem irrigada (pivô central), onde se assumiu que $\mathrm{H}$ e dT são nulos e $\mathrm{LE}=\mathrm{Rn}-\mathrm{G}$, de acordo com Bastiaanssen (2000). Já o pixel quente localiza-se numa área de solo exposto, onde se assumiu que LE é nulo, $\mathrm{H}=\mathrm{Rn}-\mathrm{G}$ e dT $=\mathrm{H} . \mathrm{r}_{\mathrm{ah}} / \rho . \mathrm{c}_{\mathrm{p}}$ (BASTIAANSSEN, 2000; BEZERRA et al., 2008). Tais valores iniciais de $\mathrm{H}$ servem, apenas, como parâmetros de entrada do processo iterativo em que é considerada a condição de estabilidade de cada pixel pela teoria da similaridade de Monin-Obukhov - L (m), segundo Allen et al. (2007a):

$$
L=-\frac{\rho c_{p} u_{*}{ }^{3} T s}{k g H}
$$

em que: $u_{*}$ é a velocidade de fricção $\left(\mathrm{m} \mathrm{s}^{-1}\right)$, Ts é a temperatura da superfície $(\mathrm{K})$, g é o módulo do campo gravitacional terrestre $\left(9,81 \mathrm{~m} \mathrm{~s}^{-2}\right)$ e $\mathrm{H}$ é o fluxo de calor sensível, obtido inicialmente considerando a condição de neutralidade.

Os valores de $\mathrm{L}$ definem as condições de estabilidade: para $\mathrm{L}<0$ (atmosfera instável); para $\mathrm{L}>0$ (atmosfera estável); e para $\mathrm{L}=0$ (atmosfera neutra). Dependendo das condições atmosféricas, os valores das correções de estabilidade para o transporte de momentum e de calor segundo Bastiaanssen (2000) e Allen et al. (2007a).

Os valores diários da evapotranspiração real diária - ETc $\left(\mathrm{mm} \mathrm{dia}^{-1}\right)$ foram obtidos a partir de extrapolação do valor instantâneo do LE (Equação 1) através da metodologia proposta por Bastiaanssen (2000):

$$
\mathrm{ETc}=0,035\left(\frac{\mathrm{LE}}{\mathrm{Rn}-\mathrm{G}}\right)\left[(1-\alpha) \mathrm{R}_{\mathrm{S} \downarrow 24 \mathrm{~h}}-110 \tau_{\mathrm{SW} \downarrow 24 \mathrm{~h}}\right]
$$

em que: $\mathrm{R}_{\mathrm{S} \downarrow 24 \mathrm{~h}}$ é a radiação solar incidente diária $\left(\mathrm{W} \mathrm{m}^{-2}\right)$, $\tau_{\mathrm{sw} \downarrow 24 \mathrm{~h}}$ é a transmissividade atmosférica diária, obtida como base na radiação solar diária observada na estação meteorológica e na radiação solar diária estimada para o topo da atmosfera nos dias estudados; e 0,035 é fator de conversão de $\mathrm{W} \mathrm{m}^{-2}$ para $\mathrm{mm} \mathrm{dia}^{-1}$. 
Por fim foi obtido o coeficiente de cultivo da cultura através da seguinte razão:

$$
\mathrm{Kc}=\frac{\mathrm{ET}}{\mathrm{ETo}}
$$

em que: ETo é a evapotranspiração de referência diária obtida pelo método de Penman-Monteith e detalhado por Allen et al. (1998).

Para construir as curvas do NDVI, da ETc e do Kc partiu-se da escolha de oito pivôs, os quais estão apresentados na Figura 1, com diferentes datas de semeadura abrangendo as cultivares Delta Penta e Delta Opal, conforme Tabela 1. Tal procedimento foi realizado para determinar um comportamento médio da cultura nas condições onde fora realizado o estudo. Na Tabela 2 estão apresentadas as datas das imagens e os respectivos Dias Após a Semeadura - DAS de cada pivô.

\section{Resultados e discussão}

A evolução temporal do NDVI do algodoeiro ao longo do seu ciclo fenológico está apresentada na Figura 2. Considerando um ciclo médio de 190 dias, os primeiros pontos referem-se às médias do NDVI dos pivôs 1 e 2 (FIG. 1), os quais foram os primeiros a serem semeados, (primeira quinzena de janeiro), conforme apresentado na Tabela 1. No período dos 70 aos $120 \mathrm{DAS}$, verifica-se que o NDVI atingiu o seu valor máximo, em torno de 0,80 . Os valores do NDVI do referido período equivale aos valores médios dos pivôs selecionados (FIG. 1). Nesse intervalo a cultura de todos os pivôs propicia a cobertura plena do solo.

O NDVI fornece informações sobre a condição nutricional e hídrica, além da capacidade fotossintética da cultura (BEZERRA; FIDELIS FILHO, 2009). Assim, tem sido demonstrado que há uma forte correlação entre o mesmo e outras variáveis da cultura, a exemplo da área foliar (BEZERRA; FIDELIS FILHO, 2009) e o coeficiente de cultura (HUNSAKER et al., 2003, 2007; LÓPEZ-URREA et al., 2009).

Tabela 1 - Data de semeadura e colheita em 2007, ciclo da cultura do algodoeiro, área plantada e cultivar do algodoeiro herbáceo dos pivôs selecionados

\begin{tabular}{cccccc}
\hline Pivô & Data de semeadura & Data de colheita & Ciclo da cultura (dias) & Área (ha) & Cultivar \\
\hline 1 & 08/janeiro & $28 /$ julho & 201 & 90 & Delta Opal \\
2 & $15 /$ janeiro & $14 /$ julho & 180 & 90 & Delta Opal \\
3 & 2 /janeiro & 07/agosto & 219 & 90 & Delta Penta \\
4 & $21 /$ janeiro & $22 /$ julho & 182 & 66 & Delta Penta \\
5 & $21 /$ janeiro & $22 /$ julho & 182 & 90 & Delta Penta \\
6 & $24 /$ janeiro & 22 e 24/agosto & 234 e 236 & 90 & Delta Penta \\
7 & $27 /$ fevereiro & $26 /$ agosto & 180 & 90 & Delta Opal \\
8 & $06 /$ março & 30/agosto & 177 & 90 & Delta Opal \\
\hline
\end{tabular}

Tabela 2 - DAS do algodoeiro dos pivôs selecionados por ocasião das datas em que foram geradas as imagens TM - Landsat 5

\begin{tabular}{ccccccccc}
\hline Pivô & $20 /$ jan & $25 /$ mar & $26 /$ abr & $12 /$ mai & $28 /$ mai & $13 /$ jun & $15 /$ jul & $31 /$ jul \\
\hline 1 & 12 & 76 & 108 & 124 & 140 & 156 & 188 & - \\
2 & 5 & 69 & 101 & 117 & 133 & 149 & 181 & - \\
3 & - & 64 & 96 & 112 & 128 & 144 & 176 & 192 \\
4 & - & 63 & 95 & 111 & 127 & 143 & 175 & - \\
5 & - & 63 & 95 & 111 & 127 & 143 & 175 & - \\
6 & - & 60 & 92 & 108 & 124 & 140 & 172 & 188 \\
7 & - & 26 & 58 & 74 & 90 & 106 & 138 & 154 \\
8 & - & 19 & 51 & 67 & 83 & 99 & 131 & 147 \\
\hline
\end{tabular}




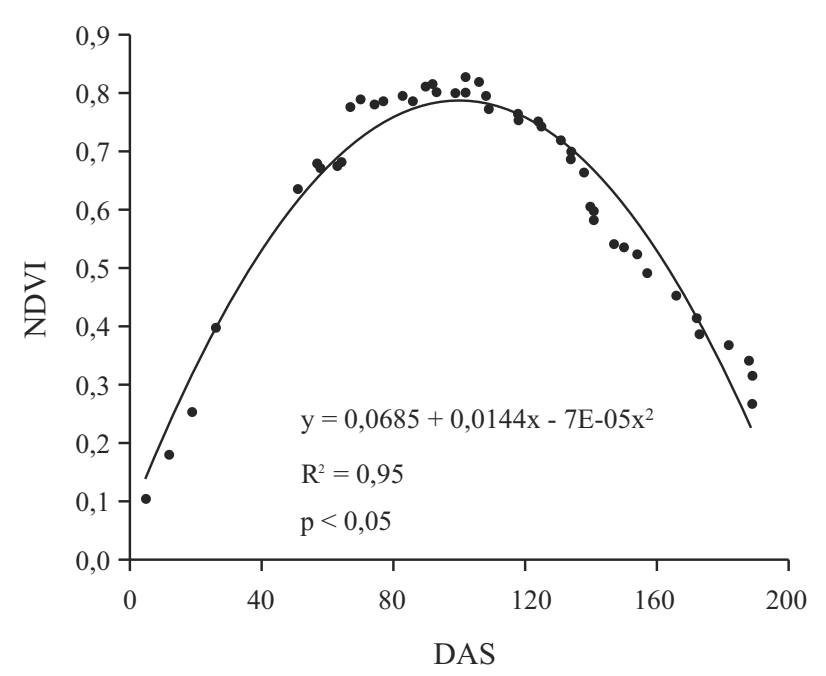

Figura 2 - Relação entre o NDVI e os dias após a semeadura

De acordo com a Figura 2, constata-se que os valores do NDVI variaram de 0,18 no início do ciclo fenológico a valores superiores a 0,80 a partir dos 70 DAS aproximadamente. Essa ascendência de valores do NDVI no período de 0 a 70 DAS corresponde ao estágio de desenvolvimento vegetativo da cultura, isto é, o período em que a cultura apresenta aumento da área foliar até atingir a plena cobertura do solo. Verifica-se também que dos 70 a 120 DAS aproximadamente, o NDVI é praticamente constante, ou seja, valores ligeiramente superiores a 0,80. Esses valores corroboram com os obtidos por Hunsaker et al. (2003), Bezerra e Fideles Filho (2009) e Montomiya et al. (2009) e são característicos do estágio de desenvolvimento reprodutivo ou estágio médio da cultura, isto é, da floração ao início da maturação. Os valores do NDVI se mostram constantes nesse estágio devido ao fato da cultura não apresentar variações na sua área foliar, visto que, o NDVI é constituído por uma combinação da refletância multiespectral e esta é sensível ao índice de área foliar e/ou fração de cobertura do solo (CHOUDHURY et al., 1994). Após 120 DAS, os valores do NDVI decresceram sucessivamente até atingir o valor de 0,30 no final do ciclo fenológico, caracterizando o período de maturação da cultura, quando ocorreram envelhecimento e morte das folhas e a consequente perda da capacidade fotossintética. Ainda na Figura 2 pode-se constatar que o coeficiente de determinação - $\mathrm{R}^{2}$ foi de aproximadamente 0,95 .
A ETo acumulada durante o ciclo fenológico da cultura do algodoeiro foi de $1.018 \mathrm{~mm}$, com mínima de 1,8 e máxima de $7,1 \mathrm{~mm}$ dia $^{-1}$. Os valores diários calculados para os dias estudados estão apresentados na Tabela 3.

As estimativas da ETc do algodoeiro herbáceo cultivares Delta Penta e Delta Opal e a curva de tendência da mesma estão apresentadas na Figura 3. A ETc variou de 0,5 a $6,7 \mathrm{~mm} \mathrm{dia}^{-1}$. Observa-se que a ETc máxima apresentada pelas cultivares estudadas foi similar aos valores apresentados por outras cultivares em diferentes áreas do semiárido brasileiro, a exemplo da Precoce 1 no oeste do estado da Paraíba (AZEVEDO et al., 1993) e a BRS-200 Marrom na região do Cariri, sul do Estado do Ceará (BEZERRA et al., 2010). Resultados semelhantes também foram obtidos por Payan e Watts (2005), Nagler et al. (2005), Conrad et al. (2007) e Bezerra et al. (2008). No período da emergência até os 6 DAS, a ETc foi inferior a $1,0 \mathrm{~mm} \mathrm{dia}^{-1}$. No período de desenvolvimento vegetativo, entre 7 e 70 DAS, a ETc variou de 1,0 a $5,0 \mathrm{~mm} \mathrm{dia}{ }^{-1}$. No estágio médio, entre 70 e $120 \mathrm{DAS}$, a ETc alcançou valores superiores a $6,0 \mathrm{~mm} \mathrm{dia}^{-1}$. Por sua vez, no período de maturação da cultura, a ETc variou de 5,5 $\mathrm{mm} \mathrm{dia}^{-1}$ aos $121 \mathrm{DAS}$ a $2,0 \mathrm{~mm} \mathrm{dia}{ }^{-1}$ no final do ciclo fenológico. A variação da ETc em cada fase fenológica apresenta compatibilidade com os resultados obtidos por Conrad et al. (2007).

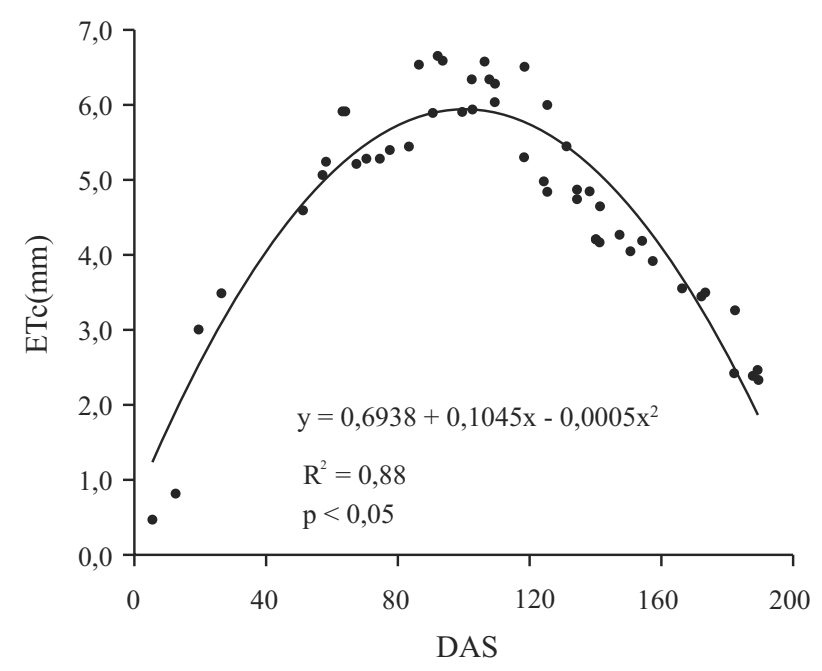

Figura 3 - Relação entre ETc e o DAS

Tabela 3 - Valores da ETo diária por ocasião das datas em que foram geradas as imagens TM - Landsat 5

\begin{tabular}{ccccccccc}
\hline Data & $20 /$ jan & $25 / \mathrm{mar}$ & $26 / \mathrm{abr}$ & $12 / \mathrm{mai}$ & $28 / \mathrm{mai}$ & $12 /$ jun & $15 /$ jul & $31 /$ jul \\
\hline $\mathrm{ET}_{0}\left(\mathrm{~mm} \mathrm{dia}^{-1}\right)$ & 6,41 & 5,38 & 4,78 & 4,81 & 4,23 & 4,47 & 4,10 & 5,42 \\
\hline
\end{tabular}


$\mathrm{O} \mathrm{Kc}$ do algodoeiro variou de 0,38 a 1,25 conforme a Figura 4 . Os respectivos valores ocorreram no estágio inicial e no estágio médio. A variação do Kc no estágio médio foi 1,11 a 1,25 , apresentando valor médio de 1,18. Esses resultados corroboram com os resultados obtidos em outras regiões potencialmente produtoras de algodão, a exemplo dos estados americanos do Texas, Califórnia, Arizona e Louisiana (GRISMER, 2002; HUNSAKER, 1999; HUNSAKER et al., 2003; HOWELL et al., 2004; KO et al., 2009), além do valor proposto pela FAO-56 (ALLEN et al., 1998) para esse estágio. Porém, foi superior aos valores obtidos no oeste do estado da Paraíba (AZEVEDO et al., 1993), na Índia (MOHAN; ARUMUGAN, 1994) e na Síria (FARAHANI et al., 2008).

A partir dos 126 DAS, fase de maturação da cultura, a curva do Kc foi sucessivamente descendente de modo a atingir o valor de 0,61 na maturação total. O valor do Kc final é semelhante aos valores obtidos por Azevedo et al. (1993) e Farahani et al. (2008), porém é inferior aos resultados obtidos por Grismer (2002), para os vales do Sacramento e São Joaquim e para os desertos da Califórnia e por Ko et al. (2009) na região de Uvalde, Texas, EUA.

Na Figura 4 está apresentada a função de ajuste do Kc com o DAS. Os dados foram ajustados a um polinômio de segundo grau com bom coeficiente de determinação. Ko et al. (2009) também ajustaram a curva do Kc do algodoeiro obtido a partir de medidas lisimétricas a um polinômio de terceiro grau com o DAS. Ajustes similares têm sido obtidos para outras culturas, a exemplo do milho e sorgo (PICCINNI et al., 2009).

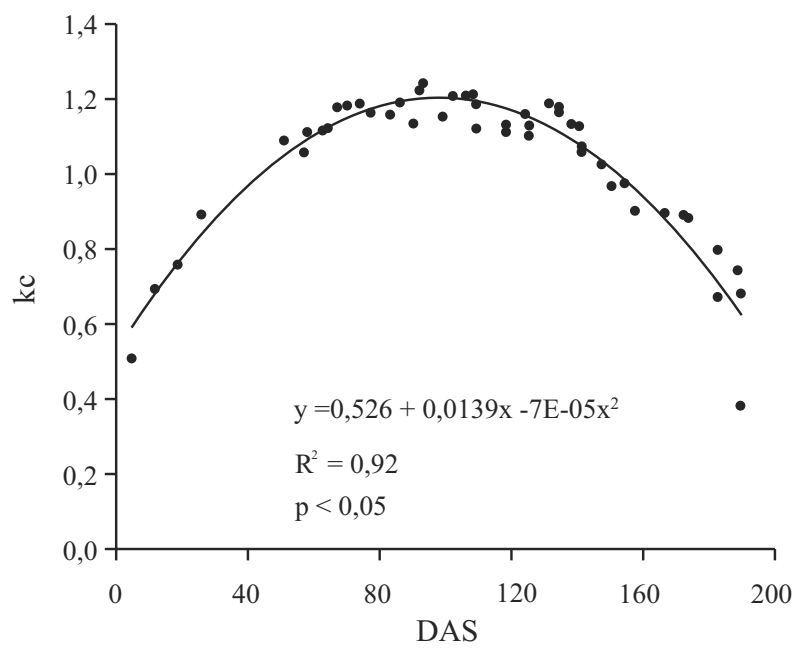

Figura 4 - Relação entre o Kc e os DAS
Na Tabela 4 estão apresentados os valores do Kc médio do algodoeiro semeados na Fazenda Busato obtidos nesta pesquisa para os estágios inicial, médio e final do mesmo através das informações oriundas das oito imagens geradas pelo TM - Landsat 5 que cobriram todo o ciclo do algodoeiro. Conforme apresentado acima, tais valores médios corroboram com outras pesquisas realizadas em todo o mundo.

Tabela 4 - Valores do Kc médio do algodoeiro semeado na Fazenda Busato obtido a partir de informações oriundas do TM - Landsat 5

\begin{tabular}{cc}
\hline Estágio & $\mathrm{Kc}$ \\
\hline Inicial & 0,65 \\
Médio & 1,18 \\
Final & 0,66 \\
\hline
\end{tabular}

\section{Conclusões}

1. O NDVI mostrou-se um bom indicador do desenvolvimento da cultura, constituindo ferramenta importante para o manejo da cultura e estimativa da produtividade;

2. A ETc diária estimada para todo o ciclo da cultura atingiu valor máximo pouco superior a $6 \mathrm{~mm} \mathrm{dia}{ }^{-1}$, demonstrando coerência com os valores apresentados pela literatura;

3. O Kc estimado para ambas as cultivares foi coerente aos valores relatados pela literatura para o algodoeiro herbáceo nas fases fenológicas consideradas.

\section{Agradecimentos}

Os autores agradecem à CAPES pela concessão de bolsas de doutorado ao primeiro e quarto autores, ao CNPq pela concessão de bolsa de Produtividade em Pesquisa ao segundo autor e de doutorado ao terceiro autor e à Fazenda Busato pela disponibilidade dos dados meteorológicos.

\section{Referências}

ALLEN, R. G. et al. Crop Evapotranspiration: Guidelines for computing crop water requirements., FAO, 1998. 300 p. (FAO Irrigation and Drainage Paper, 56).

ALLEN, R. G. et al. Prediction accuracy for projectwide evapotranspiration using crop coefficients and reference 
evapotranspiration. Journal of Irrigation Drainage Engineering, v. 131, n. 01, p. 24-36, 2005.

ALLEN, R.; TASUMI, M.; TREZZA, R. Satellite-based energy balance for mapping evapotranspiration with internalized calibration (METRIC) - Model. Journal of Irrigation and Drainage Engineering, v. 133, n. 04, p. 380-394, 2007 a.

ALLEN, R. G. et al. Satellite-based energy balance for mapping evapotranspiration with internalized calibration (METRIC) Applications. Journal of Irrigation and Drainage Engineering, v. 133, n. 04 , p. $395-405,2007$ b.

ALLEN, R. G.; PEREIRA, L. S. Estimating crop coefficients from fraction of ground cover and height. Irrigation Science, v. 28, n. 01, p. 17-34, 2009.

AZEVEDO, P. V. et al. Necessidades hídricas da cultura do algodoeiro. Pesquisa Agropecuária Brasileira, v. 28, n. 01, p. $863-870,1993$.

BASTIAANSSEN, W. G. M. et al. SEBAL model with remotely sensed data to improve water-resources management under actual field conditions. Journal of Irrigation and Drainage Engineering, v. 131, n. 01, p. 85-92, 2005.

BASTIAANSSEN, W. G. M. SEBAL - Based sensible and latent heat fluxes in the irrigated Gediz Basin, Turkey. Journal of Hydrology, v. 229, n. 01/02, p. 87-100, 2000.

BEZERRA, B. G.; FIDELES FILHO, J. Análise de crescimento da cultura do algodoeiro irrigada com águas residuárias. Revista Ciência Agronômica, v. 40, n. 03, p. 339-345, 2009.

BEZERRA, B. G.; SILVA, B. B. da; FERREIRA, N. J. Estimativa da evapotranspiração real diária utilizando-se imagens digitais TM - Landsat 5. Revista Brasileira de Meteorologia, v. 23, n. 03, p. 305-317, 2008.

BEZERRA, J. R. C. et al. Evapotranspiração e coeficiente de cultivo do algodoeiro BRS-200 Marrom, Irrigado. Revista Brasileira de Engenharia Agrícola Ambiental, v. 14, n. 06, p. $625-632,2010$

BORGES, V. P.; OLIVEIRA, A. S.; SILVA, B. B. Mapeamento e quantificação de parâmetros biofísicos e radiação líquida em área de algodoeiro irrigado. Ciência e Agrotecnologia, v. 34, n. 02 , p. $485-493,2010$.

CHOUDHURY, B. J. et al. Relations between evaporation coefficients and vegetation indices studies by model simulations. Remote Sensing of Environment, v. 50, p. 1-17, 1994.

CONRAD, C. et al. Mapping and assessing water use in a Central Asian irrigation system by utilizing MODIS remote sensing products. Irrigation and Drainage Systems, v. 21, n. 03/04, p. 197-218, 2007.

FARAHANI, H. J.; OWEIS, T. Y.; IZZI, G. Crop coefficient for drip-irrigated cotton in a Mediterranean environment. Irrigation Science, v. 26, n. 05, p. 375-383, 2008

FERERES, E.; SORIANO, M. A. Deficit irrigation for reducing agricultural water use. Journal of Experimental Botany, v. 58, n. 02, p. 147-159, 2007.
GRISMER, M. E. Regional cotton lint yield, ETc, and water value in Arizona and California. Agricultural Water Management, v. 54 , n. 03 , p. $227-242,2002$.

HOWELL, T. A. et al. Evapotranspiration of full-, deficitirrigated, and dryland cotton on the Northern Texas High Plains. Journal of Irrigation and Drainage Engineering, v. 130, n. 04 , p. $277-285,2004$.

HUNSAKER, D. J. Basal crop coefficients and water for early maturity cotton. Transactions of ASAE, v. 42, n. 04, p. 927-936, 1999.

HUNSAKER, D. J. et al. Cotton irrigation scheduling using remotely sensed and FAO-56 basal crop coefficients. Transactions of ASABE, v. 84, n. 04, p. 1395-1407, 2007.

HUNSAKER, D. J. et al. Estimating cotton evapotranspiration crop coefficients with a multispectral vegetation index. Irrigation Science, v. 22, n. 02, p. 95-104, 2003.

KO, J. et al. Determination of growth-stage-specific crop coefficients $(\mathrm{Kc})$ of cotton and wheat. Agricultural Water Management, v. 96, n. 12, p. 1691-1697, 2009.

LI, H. et al. Estimation of water consumption and crop productivity of winter wheat in North China Plain using remote sensing technology. Agricultural Water Management, v. 95, n. 11, p. 1271-1278, 2008

LÓPEZ-URREA, R. et al. Water use of spring wheat to rise water productivity. Agricultural Water Management, v. 96, n. 09, p. 1305-1310, 2009.

MOHAN, S.; ARUMUGAM, N. Crop coefficient of major crops in South India. Agricultural Water Management, v. 26, n. 01/02, p. 67-80, 1994.

MOTOMIYA, A. V.; MOLIN, J. P.; CHIAVEGATO, E. Utilização de sensor ótico ativo para detectar deficiência foliar de nitrogênio em algodoeiro. Revista Brasileira de Engenharia Agrícola e Ambiental, v. 13, n. 02, p. 137-145, 2009.

NAGLER, P. L. et al. Evapotranspiration on Western U.S. Rivers Estimated Using the Enhanced Vegetation Index from MODIS and Data from Eddy Covariance and Bowen Ratio Flux Towers. Remote Sensing of Environment, v. 97, n. 03, p. 337-351, 2005.

PAYAN, J. G.; WATTS, C. J. The use of remote sensing for estimating ET of irrigated wheat and cotton in Northwest Mexico. Irrigation and Drainage Systems, v. 19, p. 301-320, 2005.

PEREIRA, L. S. et al. Irrigation scheduling strategies for cotton to cope with water scarcity in the Fergana Valley, Central Asia. Agricultural Water Management, v. 96, n. 05, p. $732-735,2009$.

PERRY, C. et al. Increasing productivity in irrigated agriculture: Agronomic constraints and hydrological realities. Agricultural Water Management, v. 96, n. 11, p. 1517-1524, 2009.

PICCINNI, G. et al. Determination of growth-stage-specific crop coefficients $(\mathrm{Kc})$ of maize and sorghum. Agricultural Water Management, v. 96, n. 12, p. 1691-1697, 2009. 